\title{
Embedded resolutions of non necessarily normal affine toric varieties
}

\author{
Pedro Daniel González Pérez, Bernard Teissier
}

Équipe géométrie et dynamique, Institut de mathématiques, Université de Paris 7, UMR CNRS 7586, case 7012, 2, place Jussieu, 75005 Paris, France

\begin{abstract}
We give a method to construct a partial embedded resolution of a nonnecessarily normal affine toric variety $Z^{\Gamma}$ equivariantly embedded in a normal affine toric variety $Z_{\rho}$. This partial resolution is an embedded normalization inside a normal toric ambient space and a resolution of singularities of the ambient space, which always exists, provides an embedded resolution. The advantage is that this partial resolution is completely determined by the embedding $Z^{\Gamma} \subset Z_{\rho}$. As a by-product, the construction of the normalization is made without an explicit computation of the saturation of the semigroup $\Gamma$ of the toric variety (see [3]). This result is valid for a base field $k$ algebraically closed of arbitrary characteristic. To cite this article: P.D. González Pérez, B. Teissier, C. R. Acad. Sci. Paris, Ser. I 334 (2002) 379-382. (C) 2002 Académie des sciences/Éditions scientifiques et médicales Elsevier SAS

\section{Résolution plongée d'une variété torique non nécessairement normale}

Résumé Nous présentons une méthode de construction d'une résolution plongée partielle d'une variété torique affine non nécessairement normale $Z^{\Gamma}$ plongée de manière équivariante dans une variété torique affine normale $Z_{\rho}$. Cette résolution partielle est une normalisation plongée de $Z^{\Gamma}$ dans un espace ambiant torique normal et une résolution des singularités de l'espace ambiant, qui existe toujours, fournit une résolution plongée des singularités. L'avantage est que cette résolution partielle est entièrement déterminée par le plongement $Z^{\Gamma} \subset Z_{\rho}$. Une conséquence est la construction de la normalisation sans calcul de la saturation du semigroupe $\Gamma$ de la variété torique (voir [3]). Ce résultat est valide sur un corps $k$ algébriquement clos de caractéristique quelconque. Pour citer cet article:P.D. González. Pérez, B. Teissier, C. R. Acad. Sci. Paris, Ser. I 334 (2002) 379-382. () 2002 Académie des sciences/Éditions scientifiques et médicales Elsevier SAS
\end{abstract}

\section{Introduction}

The existence of a resolution of singularities of any variety was proved by Hironaka in characteristic zero and is still open in positive characteristic. For the class of toric varieties the situation is much simpler. We can find resolutions of singularities of normal toric varieties which are toric morphisms and these toric resolutions admit a completely combinatorial description independent of the characteristic of the algebraically closed base field (see [6] and [1]).

E-mail addresses: gonzalez@ math.jussieu.fr (P.D. González Pérez); teissier@ math.jussieu.fr (B. Teissier). 
In this Note we will consider an affine (non necessarily normal) toric variety $Z^{\Gamma}$ equivariantly embedded in an affine normal toric variety $Z_{\rho}$, i.e., the embedding is compatible with the actions of their tori. For instance we have monomial curves, the affine curves defined by monomial parametrizations, (see [4] for their resolutions). This Note deals with embedded toric resolutions of $Z^{\Gamma} \subset Z_{\rho}$, roughly speaking, toric resolutions of $Z_{\rho}$ that provide resolutions of $Z^{\Gamma}$. If the dimension of $Z_{\rho}$ is $>2$ there are many different toric resolutions and no generalization of the minimal toric resolution existing in the two-dimensional case. For this reason, we introduce the notion of partial resolution (inspired by some ideas of Lejeune and Reguera in a different context (see [7])). A partial resolution of $Z^{\Gamma}$ is completely characterized by a simple combinatorial property of the embedding, and it defines an embedded normalization of the variety $Z^{\Gamma}$ as a transversal section of a normal toric variety $Z_{\Sigma}$, i.e., any toric resolution of $Z_{\Sigma}$ provides also an embedded resolution of $Z^{\Gamma}$. We give an example for a monomial surface.

The results of this Note are included in a work of the first author on quasi-ordinary singularities of hypersurfaces and their embedded resolutions (see [5], Proposition 5) and in a work of the second author which deals with local uniformization of a valuation of a noetherian local integral domain by using a geometric specialization of this ring to the graded ring determined by the valuation and the tools of toric geometry (see [10], Proposition 6.4).

\section{A reminder of toric geometry}

We give some definitions and notations (see [3,2] and [8] for proofs). If $N \cong \mathbf{Z}^{d+1}$ is a lattice we denote by $M$ the dual lattice, by $N_{\mathbf{R}}$ the real vector space spanned by $N$ and by $N_{\sigma}$ the lattice spanned by $\sigma \cap N$ for $\sigma$ a cone in $N_{\mathbf{R}}$. In what follows a cone mean a rational convex polyhedral cone: the set of non negative linear combinations of vectors $a^{1}, \ldots, a^{2} \in N$. The cone $\sigma$ is strictly convex if $\sigma$ contains no linear subspace of dimension $>0$; the cone $\sigma$ is regular if the primitive integral vectors defining the 1-dimensional faces belong to a basis of the lattice $N$. We denote by $\stackrel{\circ}{\sigma}$ the relative interior of a cone $\sigma$. The dual cone $\sigma^{\vee}$ (resp. orthogonal cone $\sigma^{\perp}$ ) of $\sigma$ is the set $\left\{w \in M_{\mathbf{R}} \mid\langle w, u\rangle \geqslant 0\right.$ (resp. $\left.\left.\langle w, u\rangle=0\right) \forall u \in \sigma\right\}$. A fan $\Sigma$ is a family of strictly convex cones in $N_{\mathbf{R}}$ such that any face of such a cone is in the family and the intersection of any two of them is a face of each. The support of the fan $\Sigma$ is the set $\bigcup_{\sigma \in \Sigma} \sigma \subset N_{\mathbf{R}}$. The fan $\Sigma$ is regular if all its cones are regular; it is compatible with $\ell \subset N_{\mathbf{R}}$ if $\sigma \cap \ell \in \Sigma, \forall \sigma \in \Sigma$.

A non necessarily normal affine toric variety is of the form $Z^{\Gamma}=\operatorname{Spec} k[\Gamma]$ where $\Gamma$ is a sub-semigroup of finite type of a lattice $(\Gamma-\Gamma)$ which it generates as a group. The normalization of the variety $Z^{\Gamma}$ is obtained from the semigroup inclusion $\Gamma \rightarrow \tau \cap(\Gamma-\Gamma)$ where $\tau$ is the cone spanned by the elements of $\Gamma$ (see [6]). In particular, if $\sigma$ is a cone in the fan $\Sigma$, the semigroup $\sigma^{\vee} \cap M$ is of finite type, it spans the lattice $M$ and the variety $Z^{\sigma^{\vee} \cap M}$, which we denote also by $Z_{\sigma, N}$ or by $Z_{\sigma}$ when the lattice is clear from the context, is normal.

If $\sigma \subset \sigma^{\prime}$ are cones in the fan $\Sigma$ then we have an open immersion $Z_{\sigma} \subset Z_{\sigma^{\prime}}$; the affine varieties $Z_{\sigma}$ corresponding to cones in a fan $\Sigma$ glue up to define the toric variety $Z_{\Sigma}$. The torus $\left(k^{*}\right)^{d+1}$ is an open dense subset $Z_{\{0\}}$ of each chart $Z_{\sigma}$ acting on $Z_{\sigma}$; these actions paste into an action on $Z_{\Sigma}$ which extends the action of the torus on itself by multiplication. General toric varieties are defined by this property (see [9]). The toric varieties which can be defined using fans are precisely the normal ones (see [6]). The toric variety $Z_{\Sigma}$ is nonsingular if and only if the fan $\Sigma$ is regular. We define for each $\sigma \in \Sigma$, the closed subset $\mathbf{O}_{\sigma}$ of $Z_{\sigma}$ defined by the ideal $\left(X^{w} / w \in\left(\sigma^{\vee}-\sigma^{\perp}\right) \cap M\right)$ of $k\left[\sigma^{\vee} \cap M\right]$. The coordinate ring of $\mathbf{O}_{\sigma}$ is $k\left[\sigma^{\perp} \cap M\right]$. The map that applies a cone $\sigma$ in the fan $\Sigma$ to the set $\mathbf{O}_{\sigma} \subset Z_{\Sigma}$ is a bijection between the cones of the fan and the orbits of the torus action, each $\mathbf{O}_{\sigma}$ being the orbit of the special point $o_{\sigma}$ defined by $X^{u}\left(o_{\sigma}\right)=1$ for all $u \in \sigma^{\perp} \cap M$. We say that a fan $\Sigma^{\prime}$ is a subdivision of the fan $\Sigma$ if both fans have the same support and if every cone of $\Sigma^{\prime}$ is contained in a cone of $\Sigma$; this subdivision defines the toric modification $\pi_{\Sigma^{\prime}}: Z_{\Sigma^{\prime}} \rightarrow Z_{\Sigma}$ which is equivariant and induces an isomorphism between the tori. 


\section{Partial embedded resolution of toric varieties}

A resolution of singularities of a variety $Z$ is a smooth variety $Z^{\prime}$ with a modification $Z^{\prime} \rightarrow Z$ which is an isomorphism outside the singular locus of $Z$. Toric resolutions of singularities of normal toric varieties have a completely combinatorial description: given any fan $\Sigma$, there is a regular fan $\Sigma^{\prime}$ subdividing $\Sigma$, which contains the regular cones of $\Sigma$. The associated toric morphism $Z_{\Sigma^{\prime}} \rightarrow Z_{\Sigma}$ is then a resolution of singularities of the variety $Z_{\Sigma}$ (see [1], Theorem 5.1). We call such a fan $\Sigma^{\prime}$ a resolution of the fan $\Sigma$.

Let $\Sigma$ be any fan supported on $\rho$ defining the modification $\pi_{\Sigma}: Z_{\Sigma} \rightarrow Z_{\rho}$. Let $\mathcal{V}$ be a subvariety of $Z_{\rho}$ such that the intersection with the torus is a nonsingular dense open subset of $\mathcal{V}$. The strict transform $\mathcal{V}_{\Sigma} \subset Z_{\Sigma}$ is the subvariety of $\pi_{\Sigma}^{-1}(\mathcal{V})$ such that the restriction $\mathcal{V}_{\Sigma} \rightarrow \mathcal{V}$ is a modification. If the fan $\Sigma$ is regular, we say that the toric map $\pi_{\Sigma}: Z_{\Sigma} \rightarrow Z_{\rho}$ is an embedded pseudo-resolution of $\mathcal{V}$ if the restriction $\mathcal{V}_{\Sigma} \rightarrow \mathcal{V}$ is a modification such that the strict transform $\mathcal{V}_{\Sigma}$ is nonsingular and transversal to the orbit stratification of the exceptional locus of $Z_{\Sigma}$ (see [4]). The modification $\pi_{\Sigma}$ is an embedded resolution of $\mathcal{V}$ if in addition the restriction of the map $\pi_{\Sigma}$ to the strict transform $\mathcal{V}_{\Sigma} \rightarrow \mathcal{V}$ is an isomorphism outside the singular locus of $\mathcal{V}$ (see [4]). For a general fan $\Sigma$, we say that $\pi_{\Sigma}: Z_{\Sigma} \rightarrow Z_{\rho}$ is a partial embedded pseudo-resolution (resp. partial embedded resolution) of $\mathcal{V}$ if for any resolution $\Sigma^{\prime}$ of the fan $\Sigma$ the map $\pi_{\Sigma^{\prime}} \circ \pi_{\Sigma}$ is an embedded pseudo-resolution (resp. embedded resolution) of the variety $\mathcal{V}$. Any fan $\Sigma$ admits resolutions (see [6] and [1]), so that any partial embedded resolution in our sense extends to an embedded resolution.

Let $\Gamma$ be a semigroup as above, we will suppose that the cone $\tau$ is strictly convex so that there is a unique 0-dimensional orbit in $Z^{\Gamma}$. An embedding $Z^{\Gamma} \subset Z_{\rho}$ is equivariant if $Z_{\{0\}} \cap Z^{\Gamma}$ is the torus of $Z^{\Gamma}$ and the actions of the two tori coincide on $Z^{\Gamma}$. In this case the embedding $Z^{\Gamma} \subset Z_{\rho}$ is defined by a surjective homomorphism of semigroups:

$$
\rho^{\vee} \cap M \rightarrow \Gamma
$$

which extends to a lattice homomorphism $\psi: M \rightarrow(\Gamma-\Gamma)$. The ideal of $Z^{\Gamma}$ in $Z_{\rho}$ is generated by a finite set of binomials $\left\{X^{u_{i}}-X^{v_{i}}\right\}_{i \in I}$ such that $u_{i}, v_{i} \in \rho^{\vee} \cap M$ and $\psi\left(u_{i}\right)=\psi\left(v_{i}\right)$ (see [9], Chapter 4). The linear subspace $\ell$ orthogonal to $\operatorname{Ker}(\psi)$ is completely determined by the embedding and coincides with the intersection of the linear subspaces $\left(N_{\mathbf{R}}\right) \supset \ell_{i}:\left\langle u_{i}-v_{i}, w\right\rangle=0$ for $i \in I$, since the set of vectors $\left\{u_{i}-v_{i}\right\}_{i \in I}$ generates the kernel of $\psi$. Our main result is the following:

THEOREM 3.1. - Let $\Sigma$ be a subdivision of $\rho$ containing the cone $\sigma_{0}:=\rho \cap \ell$.

(1) The strict transform $Z_{\Sigma}^{\Gamma}$ of $Z^{\Gamma}$ by the morphism $\pi_{\Sigma}$ is contained in $Z_{\sigma_{0}}$, it is isomorphic to $Z_{\sigma_{0}, N_{\sigma_{0}}}$ and the restriction $\pi_{\Sigma} \mid Z_{\Sigma}^{\Gamma}: Z_{\Sigma}^{\Gamma} \rightarrow Z^{\Gamma}$ is the normalization map.

(2) The morphism $\pi_{\Sigma}$ is a partial embedded resolution of $Z^{\Gamma} \subset Z_{\rho}$.

\section{An example}

We give an example of this construction for a monomial surface in $\mathbf{A}_{k}^{4}$. The monomial parametrization $U_{1}=T_{1}^{2}, U_{2}=T_{2}^{2}, U_{3}=T_{1}^{3}, U_{4}=T_{1}^{7} T_{2}$ defines an equivariant embedding of a toric surface in $\mathbf{A}_{k}^{4}$, where the affine space is considered as toric variety. The semigroup homomorphism $\mathbf{Z}_{\geqslant_{0}}^{4} \rightarrow \Gamma$ corresponding to the embedding (see (1)) is defined by: $e_{1} \mapsto \gamma_{1}=(2,0), e_{2} \mapsto \gamma_{2}=(0,2), e_{3} \mapsto \gamma_{3}=(3,0), e_{4} \mapsto \gamma_{4}=$ $(7,2)$, for $\left\{e_{i}\right\}_{1}^{4}$ the canonical basis and $\left\{\gamma_{i}\right\}_{1}^{4}$ generating the semigroup $\Gamma$. The ideal of the embedding of the monomial surface is generated by $h_{1}:=U_{3}^{2}-U_{1}^{3}, h_{2}:=U_{4}^{2}-U_{1}^{4} U_{2} U_{3}^{2}$. The subdivision $\Sigma$ of $\mathbf{R}_{\geqslant 0}^{4}$ defined by the linear subspaces: $\ell_{1}: 3 u_{1}-2 u_{3}=0, \ell_{2}: 4 u_{1}+u_{2}+2 u_{3}-2 u_{4}=0$ contains the cone $\sigma_{0}:=\mathbf{R}_{\geqslant 0}^{4} \cap \ell_{1} \cap \ell_{2}$. By Theorem 3.1, the morphism $\pi_{\Sigma}$ defines a partial embedded resolution of the surface $Z^{\Gamma}$ and the open set $Z_{\sigma_{0}}$ contains the strict transform $Z_{\Sigma}^{\Gamma}$ of $Z^{\Gamma}$.

We describe now a resolution $\Sigma^{\prime}$ of the fan $\Sigma$ and we show that the map $\pi_{\Sigma^{\prime}} \circ \pi_{\Sigma}$ is an embedded resolution of the monomial surface. We do not define all the cones of the fan $\Sigma^{\prime}$ but just the regular cones 
of dimension four containing $\sigma_{0}$. The reason is that we can define a suitable fan $\Sigma^{\prime}$ in many different ways but in any case the orbits corresponding to cones in $\Sigma$ which are not contained in $\sigma_{0}$ do not intersect the strict transform of the monomial surface.

Remark 1. - In this example the cone $\sigma_{0}$ above is generated by the the vectors $a^{1}=(0,2,0,1)$ and $a^{2}=(2,0,3,7)$; it is a regular cone and therefore it must belong to $\Sigma^{\prime}$. Geometrically this means that the normalization of $Z^{\Gamma}$ is smooth. In general we have to find a regular subdivision of $\sigma_{0}$; in this two-dimensional situation there exists a unique minimal regular subdivision of $\sigma_{0}$ which corresponds geometrically to the minimal resolution of the normalization of $Z^{\Gamma}$, and this subdivision can be determined algorithmically from the pair $\left(\sigma_{0}, N_{\sigma_{0}}\right)$ (see [8]).

The intersection of $\ell_{1}$ with $\mathbf{R}_{\geqslant 0}^{4}$ is the regular cone generated by the vectors: $(2,0,3,0),(0,1,0,0)$, $(0,0,0,1)$. With respect to this basis the equation of the plane $\ell_{1} \cap \ell_{2}$ is $14 s_{1}+s_{2}-2 s_{3}=0$. This equation allows us to choose one vector in each half-space $\left(\ell_{1} \cap \ell_{2}\right)^{ \pm}$of $\ell_{1}$, such that each of them defines, with $a^{1}$ and $a^{2}$, a basis of the lattice $\ell_{1} \cap \mathbf{Z}^{4}$. We can take for instance $c^{1}:=(0,3,0,1)$ and $c^{2}=(0,1,0,1)$. The intersection of $\ell_{2}$ with $\mathbf{R}_{\geqslant 0}^{4}$ is the regular cone generated by the vectors: $(0,0,1,1),(1,0,0,2),(0,2,0,1)$. With respect to this basis the equation of the plane $\ell_{1} \cap \ell_{2}$ is $2 t_{1}-3 t_{2}=0$. This equation allows us to choose one vector in each half-space $\left(\ell_{1} \cap \ell_{2}\right)^{ \pm}$of $\ell_{2}$, such that each of them defines, with $a^{1}$ and $a^{2}$, a basis of the lattice $\ell_{2} \cap \mathbf{Z}^{4}$. We can take for instance $d^{1}:=(1,0,2,4)$ and $d^{2}=(1,0,1,3)$. We can verify that the cones $\theta_{i j}:=\left\langle a^{1}, a^{2}, c^{i}, d^{j}\right\rangle$ are regular for $i, j=1,2$ and they are part of a resolution $\Sigma^{\prime}$ of the fan $\Sigma$. The toric morphism $\pi_{\Sigma^{\prime}} \circ \pi_{\Sigma}$ is defined on the chart $Z_{\theta_{11}}$ by:

$$
U_{1}=W_{2}^{2} W_{4}, \quad U_{2}=W_{1}^{2} W_{3}^{3}, \quad U_{3}=W_{2}^{3} W_{4}^{2}, \quad U_{4}=W^{1} W_{2}^{7} W_{3} W_{4}^{4} .
$$

The total transform of $h_{1}=0$ (resp. of $h_{2}=0$ ) on this chart is defined by $W_{2}^{6} W_{4}^{3}\left(W_{4}-1\right)=0$ (resp. by $W_{1}^{2} W_{2}^{14} W_{3}^{2} W_{4}^{8}\left(1-W_{3}\right)=0$ ). The strict transform of the monomial surface is defined in this chart by $W_{4}=1$ and $W_{3}=1$. We remark immediately that it is smooth and transversal to the canonical stratification of the exceptional divisor (defined by $W_{i}=0$, for $i=1, \ldots, 4$ in this chart).

Acknowledgements. The first author is supported by a Marie Curie Fellowship of the European Community programme "Improving Human Research Potential" under contract number HPMF-CT-2000-00877.

\section{References}

[1] D. Cox, Toric varieties and toric resolutions, in: Resolution of Singularities, Progr. Math., Vol. 181, Birkhäuser, 2000, pp. 259-283.

[2] G. Ewald, Combinatorial Convexity and Algebraic Geometry, Springer-Verlag, 1996.

[3] W. Fulton, Introduction to Toric Varieties, Ann. of Math. Stud., Vol. 131, Princeton University Press, 1993.

[4] R. Goldin, B. Teissier, Resolving singularities of plane analytic branches with one toric morphism, in: Resolution of Singularities, Progr. Math., Vol. 181, Birkhäuser, 2000, pp. 315-340.

[5] P.D. González Pérez, Toric embedded resolutions of quasi-ordinary hypersurfaces, Preprint, 2001.

[6] G. Kempf, F. Knudsen, D. Mumford, B. St Donat, Toroidal Embeddings, Springer Lecture Notes in Math., Vol. 339, Springer-Verlag, 1973.

[7] M. Lejeune-Jalabert, A. Reguera López, Arcs and wedges on sandwiched surface singularities, Amer. J. Math. 121 (6) (1999) 1191-1213.

[8] T. Oda, Convex Bodies and Algebraic Geometry, Ann. of Math. Stud., Vol. 131, Springer-Verlag, 1988.

[9] B. Sturmfels, Gröbner Bases and Convex Polytopes, University Lecture Series, Vol. 8, American Mathematical Society, 1996.

[10] B. Teissier, Valuations, deformations and toric geometry, in preparation. 